\title{
Pleural empyema caused by incarceration and perforation of the stomach wall discovered several years later after thoracic trauma
}

\author{
Szymon Smoliński, Mariusz Kasprzyk, Piotr Gabryel, Cezary Piwkowski
}

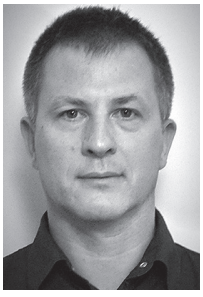

Department of Thoracic Surgery, Wielkopolskie Centrum Pulmonologii i Torakochirurgii, Poznan, Poland

Kardiochirurgia i Torakochirurgia Polska 2017; 14 (2): 143-145

Pleural empyema occurs most often as a complication of pneumonitis, a complication of diagnostic or surgical procedures within the chest, or a consequence of chest trauma [1]. Performing imaging examinations (chest X-ray, pleural ultrasound, and chest computed tomography (CT)) and thoracocentesis enables quick diagnosis and implementation of proper therapy. During the early stage of empyema development (exudative phase), the treatment is conservative and consists in draining the pleural cavity and administering antibiotics. When diagnosis is delayed (and achieved during the fibrinopurulent phase or the organizing phase), surgical treatment often becomes a necessity. In chest trauma patients, empyema usually develops due to the infection of a pleural hematoma. However, it should be remembered that, in post-traumatic patients, diaphragmatic injury must be excluded before the start of treatment. Differentiating pleural empyema and post-traumatic diaphragmatic injury is difficult, especially if the diagnostic process is delayed; at the same time, a hasty decision to drain the pleural cavity may result in tragic complications [2]. One should also keep in mind the possibility of abdominal organ incarceration in the defect of the injured diaphragm, which usually manifests with tumultuous symptoms occurring shortly after the injury. This article presents a rare case of pleural empyema resulting from the rupture of the diaphragmatic dome with stomach incarceration and perforation, diagnosed several years after the injury.

The 35-year-old male patient was admitted to the Department of Thoracic Surgery in January 2012 with suspicion of left pleural empyema. Months earlier, after vomiting, he started to experience moderate pain in the left side of the chest and epigastrium. He received analgesics and muscle relaxants. Approximately 8 days before admission, the patient's condition deteriorated, and he started to experience dyspnea, fever $\left(38.5^{\circ} \mathrm{C}\right)$, fatigue, and loss of appetite. Four years earlier, the patient had suffered a blunt trauma to the chest during a motorcycleaccident, but was not hospitalized at the time. Laboratory investigation revealed anemization $(\mathrm{Hb}$ : $8.1 \mathrm{~g} / \mathrm{dl}$; Hct: $29 \%$ ), leukocytosis (WBC: $16.4 \mathrm{G} / \mathrm{l}$ ), and a high level of C-reactive protein (CRP) (246 g/dl). Chest X-ray re- vealed only left-sided pneumothorax by demonstrating an air-fluid level in the left pleural cavity. Left pleurocentesis was performed, obtaining purulent content. Left pleural empyema was diagnosed; a drain was introduced through the $5^{\text {th }}$ intercostal space in the midaxillary line, obtaining $2200 \mathrm{ml}$ of purulent content; empirical and later targeted antibiotic treatment was introduced, resulting in evident clinical improvement. The Enterobacter cloacae (ESBL) strain was cultured from samples of the pleural fluid. Over the next 3 days, the daily volume of drained fluid remained at the level of $600 \mathrm{ml}$. On the $5^{\text {th }}$ day of drainage, the volume increased to $1000 \mathrm{ml}$, and its appearance was observed to change after meals. After the oral administration of methylene blue, its presence was confirmed in the pleural drain. Chest CT with oral Uropoline contrast demonstrated that the contrast agent leaked into the left pleural cavity from the stomach, which had been displaced into the chest (Fig. 1). The patient was diagnosed with a gas-

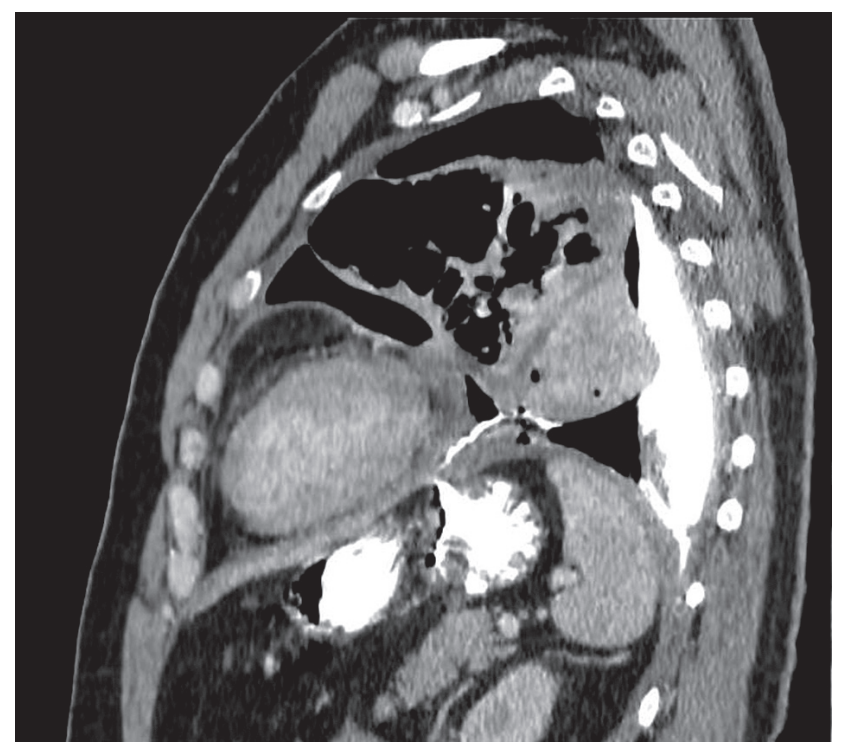

Fig. 1. Chest computed tomography (CT) with oral Uropoline contrast, showing the perforated fundus of the stomach displaced into the left pleural cavity

Address for correspondence: Szymon Smoliński MD, Department of Thoracic Surgery, Wielkopolskie Centrum Pulmonologii i Torakochirurgii, 62 Szamarzewskiego St, 60-569 Poznan, Poland, phone: +48 606667 422, fax: +48 606667 422, e-mail: szymon.smolinski.mail@gmail.com Received: 21.07.2016, accepted: 16.08.2016. 


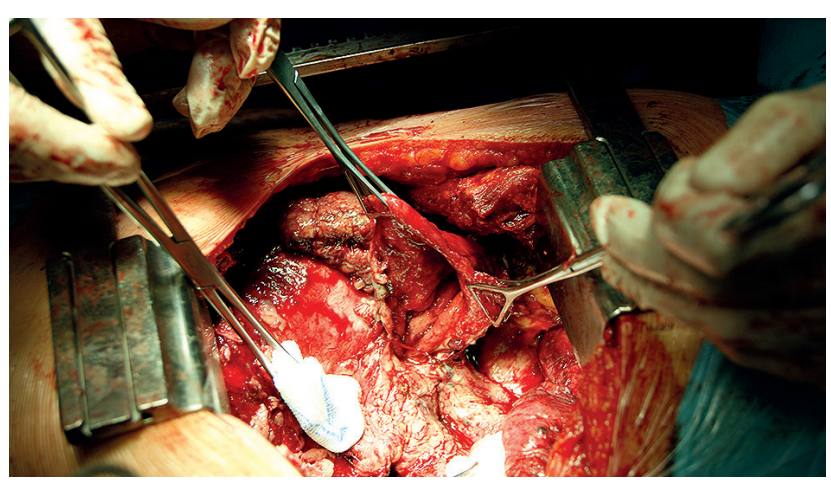

Fig. 2. Intraoperative image of the extensively perforated wall of the fundus incarcerated in the ruptured dome of the diaphragm

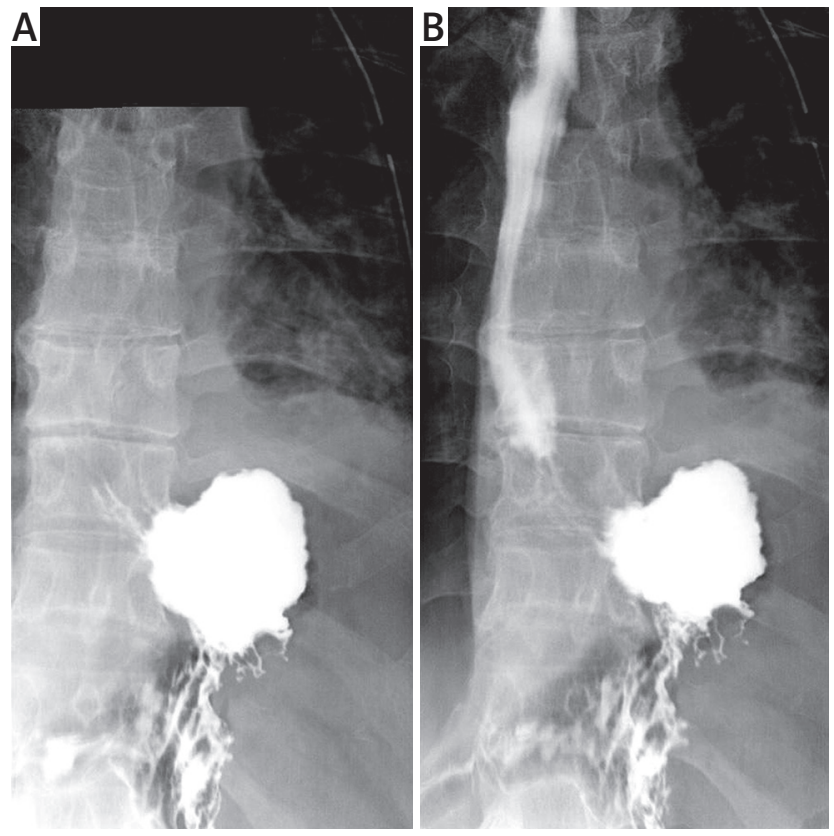

Fig. 4. Contrast examination of the upper segment of the gastrointestinal tract ( $8^{\text {th }}$ postoperative day)

tropleural fistula and scheduled for surgical treatment. Posterolateral thoracotomy was performed under the $7^{\text {th }}$ left rib. Extensive perforation of the fundus of the stomach incarcerated in the ruptured diaphragm and concomitant pleural empyema were confirmed intraoperatively (Fig. 2). After the lung was decorticated and the walls of the empyema cavity were excised, the stomach, displaced into the chest through a defect in the diaphragm (diameter: $4 \mathrm{~cm}$ ), was uncovered; its wall was partially ischemic and perforated over the length of $20 \mathrm{~cm}$. The wall of the stomach was freed from the mediastinum, lung, and diaphragm margins, and an additional incision was made in the diaphragm. Using a linear stapler, part of the fundus and body of the stomach was excised together with the fistula located in the margin of the healthy wall, and the suture line was covered with mesh (Fig. 3). The stomach was repositioned into the peritoneal cavity, and the diaphragm was sutured with a double line of nonabsorbable continuous suture. After the procedure, drains were left in the pleural cavity and the peritoneal cavity. The peritoneal drain was

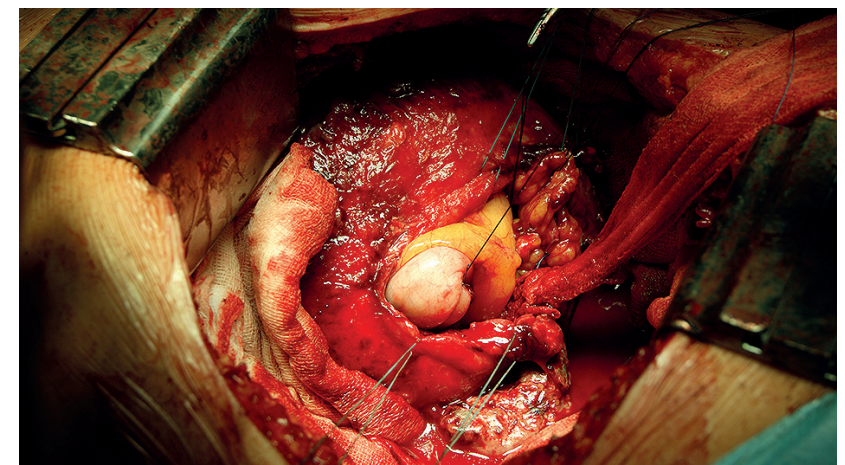

Fig. 3. Intraoperative image: partial resection of the stomach, the staple line covered with mesh

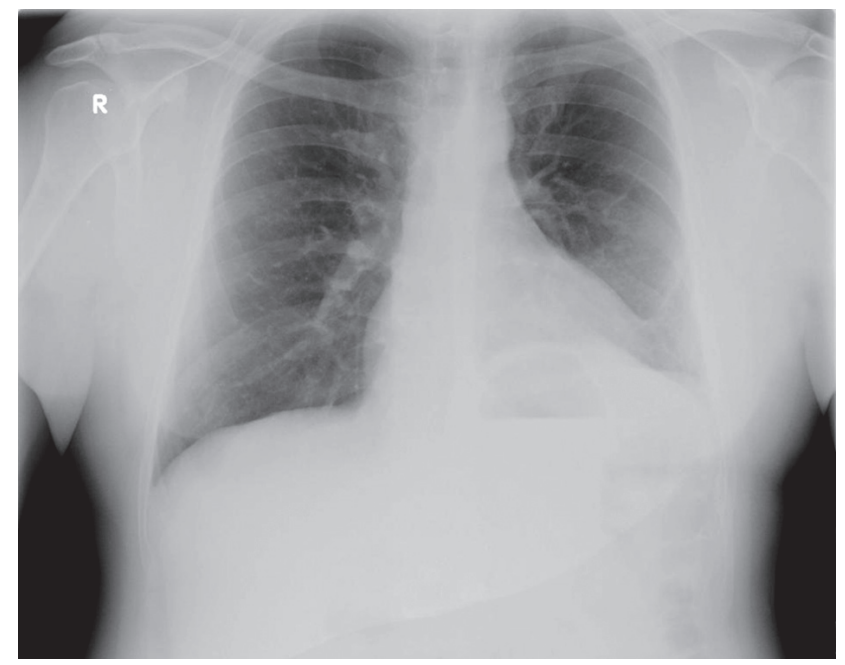

Fig. 5. Follow-up chest $\mathrm{X}$-ray 30 days after the surgery

removed on the $3^{\text {rd }}$ postoperative day, and the pleural drain - on the $5^{\text {th }}$ postoperative day. The wound healed normally. Due to fever persisting after the procedure, targeted antibiotic therapy was continued until the $14^{\text {th }}$ postoperative day. On the $7^{\text {th }}$ day after the procedure, a contrast examination of the upper segment of the gastrointestinal tract was performed; no contrast leakage was observed outside the lumen of the gastrointestinal tract (Fig. 4). On the $8^{\text {th }}$ day, oral feeding was started. The patient was discharged in good general condition on the $17^{\text {th }}$ postoperative day. Follow-up X-ray examinations, performed 4 weeks and 3 months after discharge at an outpatient thoracic surgery clinic, did not demonstrate any worrying symptoms or abnormalities (Fig. 5).

Post-traumatic diaphragm rupture occurs in $0.8 \%$ to $1.6 \%$ of patients with blunt chest trauma and in $15 \%$ to $59 \%$ of patients with penetrating thoraco-abdominal injuries [2, 3]. This incidence may be underestimated as some of the patients with post-traumatic diaphragm rupture die from other injuries before reaching the hospital; on the other hand, some injured patients without intense symptoms do not seek medical assistance or do not undergo in-depth diagnostic evaluation. The rupture is more likely to occur on the left side, which stems from the protective role of the liver on the right side and the presence of physiologi- 
cal points of decreased resistance within the left dome of the diaphragm $[2,4]$. Spontaneous healing of diaphragmatic rupture is very rare due to the contracting muscles of the injured diaphragm and the displacement of abdominal organs into the pleural cavity resulting from the pressure gradient. Extensive rupture of the left diaphragmatic dome leads to immediate displacement of abdominal organs into the thoracic cavity and, often, causes symptoms of acute respiratory insufficiency; notwithstanding, early diagnosis after trauma may be impeded by ailments associated with concomitant injuries [5]. Symptoms of diaphragmatic rupture (such as abdominal and chest pain, shoulder pain, or dyspnea after meals or when leaning forwards) can occur with various intensity, and in many cases their appearance is delayed $[6,7]$. If the diaphragmatic defect is small, abdominal organ displacement into the pleural cavity happens gradually over months or years, manifesting by progressive impairment of aerobic fitness and increasingly higher positions of the diaphragmatic dome on successive radiograms [8]. In the presented case, the injury occurred on the left side; despite the passing of several years, it did not heal on its own, and life-threatening complications developed. The diaphragmatic rupture was not large enough to cause a massive displacement of abdominal organs into the chest, which would have resulted in intense symptoms, likely prompting additional diagnostic examinations in the period immediately following the injury. Instead, over the period of several months, the fundus of the stomach was slowly displaced through the rupture into the left pleural cavity because of the pressure difference between the pleural cavity and the peritoneal cavity. Compression by the ruptured diaphragm caused chronic ischemia of the fundus wall; subsequently, vomiting resulted in a sudden pressure rise in the stomach and the perforation of its ischemic wall. The patient did not, however, manifest symptoms of gastrointestinal tract obstruction, which, according to Carter, appear within 3 years from the trauma in nearly $85 \%$ of patients with undiagnosed diaphragm injury [4]. Only part of the fundus was displaced into the chest, without impeding the passage of food, but the opening in the diaphragm was narrow enough to cause significant compression and ischemia of the stomach's wall. The clinical presentation of the perforation was also unusual. No acute symptoms of gastrointestinal tract perforation were observed because the site of the gastric fistula was separated from the peritoneal cavity and the free pleural cavity by massive post-traumatic adhesions. Pleural inflammation, caused be necrosis in the stomach's wall and the leakage of food content into the pleural cavity, led to the development of pleural empyema; it was the symptoms of the latter condition that were the immediate cause of the patient's hospitalization. After the pleural cavity was drained, the change in the character of the drained fluid after meals became a source of concern. Diagnosis of gastrointestinal tract perforation into the pleural cavity was confirmed by administering oral methylene blue and performing chest CT with oral contrast. In the described scenario, iatrogenic stomach injury should be taken into ac- count when the drain is introduced into the pleural cavity. However, in our patient, the drain was introduced into the pleural cavity through a relatively high position ( $5^{\text {th }}$ intercostal space), without the use of a trocar, and after a palpation examination (with a finger introduced into the pleural cavity); no ailments from the digestive system occurred immediately after the drainage. The described case should also be differentiated with spontaneous esophageal rupture (Boerhaave's syndrome). However, the symptoms of this syndrome are tumultuous and occur suddenly after persistent vomiting; the perforation occurs in the distal segment of the esophagus; and the patient's condition deteriorates rapidly due to mediastinitis and the development of sepsis.

According to the literature, mortality among patients operated on due to the necrosis of digestive tract elements displaced into the chest reaches $80 \%[6,9]$. It should be stressed that, in the described case, the performed surgical treatment led to complete recovery from the gastric perforation despite the significantly delayed diagnosis and the development of pleural empyema. Surgical access by means of thoracotomy was definitely warranted. The presence of pleural empyema with massive adhesions between the displaced stomach and the mediastinum and lung would have precluded safe resection and retracting the stomach into the peritoneal cavity by means of laparotomy. Laparotomy is recommended only in the early posttraumatic period (first 2-4 days after the injury), primarily because it can be used to treat other injuries within the abdominal cavity [10]. The performance of thoracotomy also enabled the surgeons to perform lung decortication and excise the wall of the empyema sac, thus facilitating healing in the inflamed pleural cavity. The relatively small opening in the diaphragm was treated with primary suturing of the diaphragm margins, without the need for synthetic materials, which could have posed an additional risk of chronic infection in the purulent environment.

\section{Disclosure}

Authors report no conflict of interest.

\section{References}

1. Ris HB, Pezzetta E, Krueger E, Lardinois D. Surgical treatment of pleural infections: the surgeon's point of view. European Respiratory Society Monograph 2004; 29: 181-198.

2. Shah R, Sabanathan S, Mearns AJ, Choudhury AK. Traumatic rupture of diaphragm. Ann Thorac Surg 1995; 60: 1444-1449.

3. Ahmed N, Jones D. Video-assisted thoracic surgery: state of the art in trauma care. Injury 2004; 35: 479-489.

4. Carter BN, Giuseffi J, Felson B. Traumatic diaphragmatic hernia. Am J Roentgenol Radium Ther 1951; 65: 56-72.

5. Hood RM. Traumatic diaphragmatic hernia. Ann Thorac Surg 1971; 12: 311-324.

6. Hanna WC, Ferri LE, Fata P, Razek T, Mulder DS. The current status of traumatic diaphragmatic injury: lesson learned from 105 patients over 13 years. Ann Thorac Surg 2008; 85: 1044-1048.

7. Blitz M, Louie MB. Chronic traumatic diaphragmatic hernia. Thorac Surg Clin 2009; 19: 491-500.

8. Sutton JP, Carlisle RB, Stephenson SE Jr. Traumatic diaphragmatic hernia: a review of 25 cases. Ann Thorac Surg 1967; 3: 137-157.

9. Hegarty MM, Bryer JV, Angorn IB, Baker LW. Delayed presentation of traumatic hernia. Ann Surg 1978; 188: 229-233.

10. Scharff JR, Naunheim KS. Traumatic diaphragmatic injuries. Thorac Surg Clin 2007; 17: 81-85. 